\title{
Contrast in greyscales of graphs
}

\author{
Natalia de Castro ${ }^{1}$ (D) María A. Garrido-Vizuete ${ }^{1}$ (D) - Rafael Robles ${ }^{1}$ (D) \\ María Trinidad Villar-Liñán ${ }^{2}$
}

Published online: 28 January 2020

(c) Springer Science+Business Media, LLC, part of Springer Nature 2020

\begin{abstract}
In this paper, we present the notion of greyscale of a graph, as a colouring of its vertices that uses colours from the continuous spectrum $[0,1]$. Any greyscale induces another colouring by assigning to each edge the non-negative difference between the colours of its vertices. These edge colours are ordered in lexicographical increasing order and make up the contrast vector of the greyscale. The aim of the contrast problem for the given graph is to find the maximum contrast vector and the greyscales that give rise to it, namely the maximum contrast greyscales. The NP-completeness of this problem is stated by means of a functional relation between the chromatic number and the first component of the maximum contrast vector, named the lightest tone. Thus, we introduce the notion of lightest tone as a new invariant of the graph. The underlying structure of values of maximum contrast greyscales is addressed and we prove that they are linked by rational number sets, which are algorithmically determined. The restricted maximum contrast problem, that is, greyscales with prefixed extreme tones, is also defined and solved in polynomial time for different families of bipartite graphs.
\end{abstract}

Keywords Graph colouring · Greyscale · Maximum contrast · NP-completeness

Natalia de Castro

natalia@us.es

María A. Garrido-Vizuete

vizuete@us.es

Rafael Robles

rafarob@us.es

María Trinidad Villar-Liñán

villar@us.es

1 Dpto. de Matemática Aplicada I, Universidad de Sevilla, Avda. Reina Mercedes s/n, 41012

Seville, Spain

2 Dpto. de Geometría y Topología, Facultad de Matemáticas, Universidad de Sevilla, Avda. Reina Mercedes s/n, 41012 Seville, Spain 
Mathematics Subject Classification Primary 05C - 68R; Secondary 05C15 - 05C85 . 90C47

\section{Introduction}

Graph colouring is one of the most studied problems in combinatorial optimization because it has a wide range of applications such as the wiring of printed circuits (Cheng et al. 1992), resource allocation (Kasi et al. 2019; Woo et al. 1991), frequency assignment problems (Aardal et al. 2007; Chaudhry et al. 2016; Griggs and Král 2009; Orden et al. 2016), wi-fi channel assignment (Orden et al. 2018, 2019), several kinds of scheduling problems (Marx 2004; Ganguli and Roy 2017), or computer register allocation (Chaitin et al. 1981; Demange et al. 2015).

A variety of combinatorial optimization problems on graphs can be formulated similarly in the following way. Given a graph $G(V, E)$, a mapping $f: V \longrightarrow \mathbb{Z}$ is defined and it induces a new mapping $\hat{f}: E \longrightarrow \mathbb{Z}$ by $\widehat{f}(e)=|f(u)-f(v)|$ for every $e=\{u, v\} \in E$. Then an optimization problem is formulated from several key elements: mappings $f$ belonging to a subset $S$, the image of $V$ by $f$ and the image of $E$ by $\widehat{f}$. In particular, the classic graph colouring problem, that is, colouring the vertices of $G$ with as few colours as possible so that adjacent vertices always have different colours, can be stated in these terms as follows:

$$
\chi(G)=\min _{f \in S}|\operatorname{Im}(f)| \text { where } S=\{f: V \rightarrow \mathbb{Z} \text { such that } 0 \notin \widehat{f}(E)\}
$$

It is well known that this minimum number $\chi(G)$ is called the chromatic number of the graph $G$ and that its computation is an NP-hard problem (Karp 1972).

In this paper, we present the new notion of greyscale of a graph, as a colouring of its vertices that uses colours from the continuous spectrum [0, 1]. Any greyscale induces an edge colouring by assigning to each edge the non-negative difference between the colours of its vertices. These edge colours are ordered in lexicographical increasing order and make up the contrast vector of the greyscale. We pose the problem of finding a maximum contrast vector and the greyscales that give rise to it, namely the maximum contrast greyscales.

Throughout Sect. 3, the close relation between our problem and the classic graph colouring problem is brought to light. In particular, each maximum contrast greyscale of $G$ provides a coloring of $G$ with precisely $\chi(G)$ colours, that is, a solution of the graph colouring problem.

It must be stressed that the classic graph colouring problem takes into account the number of colours used but not which they are. However, there are several works related to map colouring for which the nature of the colours is essential, whereas the number of them is fixed. The maximum differential graph colouring problem (Hu et al. 2011), or equivalently the antibandwidth problem (Leung 1984), colours the vertices of the graph in order to maximize the smallest colour difference between adjacent vertices and using all the colours $1,2, \ldots,|V|$. Under the above formulation, these problems are posed as follows: 
$\max _{f \in S} \min \widehat{f}(E)$ for $S=\{f: V \rightarrow \mathbb{Z}$ such that $\operatorname{Im}(f)=\{1,2, \ldots,|V|\}\}$,

and therefore the complementary optimization case, the bandwidth problem, is given by

$\min _{f \in S} \max \widehat{f}(E)$ for $S=\{f: V \rightarrow \mathbb{Z}$ such that $\operatorname{Im}(f)=\{1,2, \ldots,|V|\}\}$.

Note that these problems are concerned with the optimization of a scalar function and mappings that take values within a discrete spectrum. Dillencourt et al. (2007) studied a variation of the differential graph colouring problem under the assumption that all colours in the colour spectrum are available, more precisely, the space of colours is a three-dimensional subset of $\mathbb{R}^{3}$. This makes the problem continuous rather than discrete, since the mapping $f$ has image in $\mathbb{R}^{3}$ [see Dillencourt et al. (2007) for details].

Other colouring problems are included in the examination scheduling problem category [see for instance Bullnheimer (1998)]. This problem consists of assigning a number of exams to a number of potential time periods or slots within the examination period, taking into account that no student can take two or more exams at the same time. The graph $G$ associated to the examination scheduling problem has a vertex for each exam and two vertices are adjacent whenever there is at least one student taking their corresponding exams. This way, the chromatic number of $G$ provides the minimum number of slots needed to generate an examination period schedule.

Regarding this problem, if colours $t \in[0,1]$ are considered to be the time as a continuous variable, a greyscale provides a colouring with the property that the difference of colours between any pair of adjacent vertices represents the slot between any two exams. This way, a maximum contrast greyscale generates a solution of the examination scheduling problem with maximum difference of slot for adjacent vertices. Therefore, an optimal time period distribution that benefits every exam candidate is guaranteed.

When a weight $w_{i}$ is associated to each colour $i$ in a proper colouring of $G$, and the sum of those colour weights is minimized, the optimization problem is known to be the minimum sum colouring problem, whose applications to scheduling problems and resource allocations were recently developed [see Lecat et al. (2017) and the references therein].

In all these approaches the colouring functions are considered to be scalar mappings. In our work, we present an alternative method that can solve colouring problems focusing in the optimization of a vector that measures the differences of colours between adjacent vertices in the graph.

Thus, a new concept related to the colouring of a graph $G=G(V, E)$ is accurately introduced in Sect. 2: the contrast associated to a greyscale of $G$. Namely, given the graph $G$, a greyscale is a mapping that associates a value from the interval $[0,1]$ to each vertex $v \in V$. This assignment can be understood as an extension of the colouring of the vertices of $G$ with grey tones. For the contrast problem, the objective is to maximize the difference of tones of grey between extremes of any edge. 

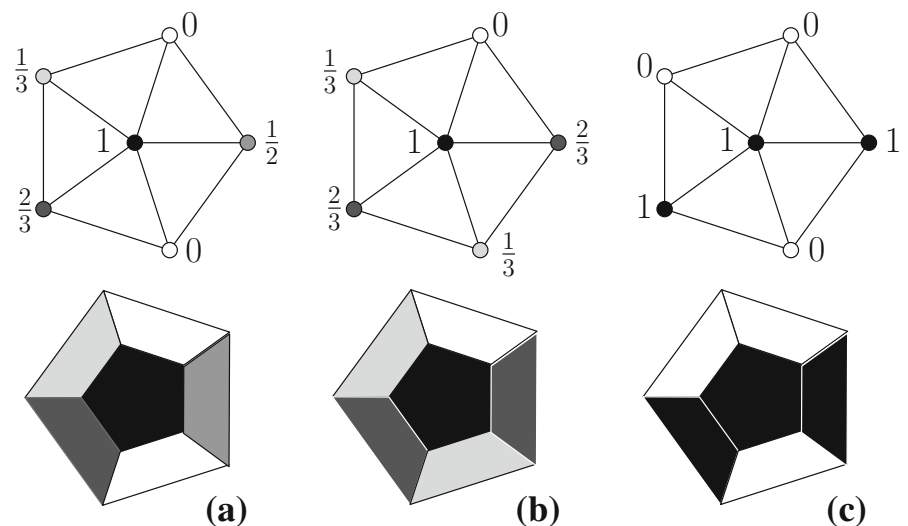

(a)

(b)

(c)

Fig. 1 A comparison of three greyscales for the wheel according to their contrasts

When the goal is to minimize the difference of tones of grey between extremes of any edge, another new problem arises: the gradation problem which is deeply studied in a work by the same authors of this paper in de Castro et al. (2018).

By using a vectorial function which allocates grey tones in an equitable manner, we propose a novel way to maximize the colour differences in the graph. More specifically, we are not interested in maximizing the total amount of "contrast" (here contrast means the colour differences between adjacent vertices) neither maximizing the minimum contrast (scalar function) but maximizing the vector whose components represent local contrast of adjacent vertices, in ascending order. Figure 1 visually shows the goodness of our vectorial optimization versus a scalar optimization, based on the total amount of contrast on edges or alternatively on maximin criterium.

Let us illustrate this fact by considering three colourings of the faces of the map in Fig. 1. We construct its dual graph avoiding the external face in such a way that the resulting graph is the wheel. Three greyscales for the wheel are presented. On one hand, according to the total amount of contrast criterium, Fig. 1c is an optimal solution (with total amount of contrast equal to 7 vs. 35/6 and 5 in Fig. 1a, b, respectively). However, in (c) there are some adjacent faces having no contrast. On the other hand, (a) and (b) are solutions under the scalar maximin criterium (both of them have minimum contrast on edges equal to 1/3). Nevertheless, only (a) is an optimal solution under our vectorial criterium. In (a), it is not difficult to check that every pair of adjacent faces has the maximum possible contrast. This way, the main advantage of our proposal lies in the possibility of obtaining a local distribution of the maximum contrast for every vertex in the graph.

We want to emphasize that a maximum contrast greyscale can be used to colour a map with greytones in such a manner that every pair of adjacent regions are coloured with best visual contrast, that is an optimal way for a black and white illustration with a quite good quality. Thus, the solutions to our problem contribute to providing solutions to the map colouring problem (Bekos et al. 2017).

In this work, we deal with proper vertex colouring but also with edge colourings of graphs which are not proper in general. Let us remark that although there exists 
a vast literature on different perspectives of the edge colouring problem (Borghini et al. 2018; Orden et al. 2018, 2016, 2019), and the references therein, the approach presented here is novel to the best of our knowledge.

Outline of the paper In Sect. 2 the necessary definitions about the contrast problem on graphs are established. Section 3 contains our first main result, Theorem 1, stating the NP-completeness of the maximum contrast problem by means of a close relation between the classical colouring problem and the contrast problem. In fact, we introduce a new invariant of the graph, the lightest tone, and establish a functional relation with the chromatic number of the same graph in Theorem 2. The main results of Sect. 4 are addressed to support the definition of maximum contrast vector. Actually, this maximum exists as it is proven in Theorem 4 and as a consequence, it assures that the notion of maximum contrast vector is well defined. Besides, the set of possible values for a maximum contrast greyscale can be bounded by a finite rational set determined algorithmically as it is collected in Theorem 5. The underlying structure of this set of values is discovered to be grouped on linked sequences named $h$-minimum enchained sets in Theorem 3.

In Sect. 5 we also define restricted versions of the original problem when the grey tones 0 or 1 of some vertices are a priori known and the aim is to obtain the maximum contrast vector preserving such fixed grey tones. For the 2-chromatic graphs family, the set of values of the maximum contrast greyscale for the restricted problem is characterized in Theorem 6. Besides, the restricted maximum contrast problem is solved in polynomial time for the family of bipartite complete graphs and for subdivisions of star graphs as is shown in Theorems 7 and 9, respectively. Other contribution of our work is Theorem 8 which collects the solutions of the restricted problem on particular cases for trees. The last section contains a brief review of open problems and future works.

\section{Preliminaries}

This section is devoted to establishing the basic concepts about contrast on graphs and formulating the problems to be studied in this paper. Throughout this paper, a graph is finite, undirected and simple and is denoted by $G(V, E)$, where $V$ and $E$ are its vertex-set and edge-set, respectively. The number of elements of $V$ and $E$ are denoted by $n$ and $m$, respectively. Let $N(v)$ denote the set of neighbours of the vertex $v$ and let $\operatorname{deg}(v)$ denote the degree of $v$, that is the cardinal of $N(v)$. For further terminology we follow Harary (1969).

Given a graph $G(V, E)$, a greyscale $f$ of $G$ is a mapping of $V$ to the interval $[0,1]$ such that values 0 and 1 belong to $\operatorname{Im}(f)$. For each vertex $v$ of $G$, we call $f(v)$ the grey tone of $v$, or more generally, the colour of $v$. Notice that two adjacent vertices may have mapped to the same grey tone. In particular, values 0 and 1 are called the extreme tones or white and black colours, respectively.

Associated to each greyscale $f$ of the graph $G(V, E)$, the mapping $\widehat{f}$ is defined on $E$ to the interval $[0,1]$ as $\widehat{f}(e)=|f(u)-f(v)|$ for each $e=\{u, v\} \in E$ and it represents the gap or increase between the grey tones of vertices $u$ and $v$. The value 


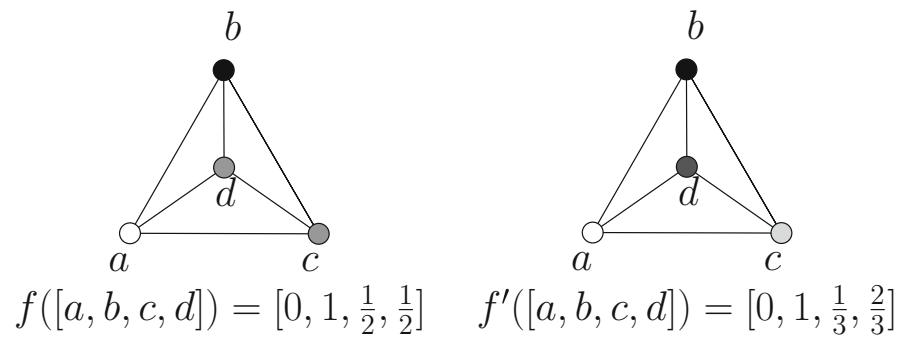

Fig. 2 Two greyscales $f$ and $f^{\prime}$ of the graph $K_{4}$

$\widehat{f}(e)$ is also said to be the grey tone of the edge $e$. Thus, we deal with coloured vertices and edges by $f$ and $\widehat{f}$, respectively.

The contrast vector associated to the greyscale $f$ of $G$ is defined to be $\operatorname{cont}(G, f)=$ $\left(\widehat{f}\left(e_{1}\right), \widehat{f}\left(e_{2}\right), \ldots, \widehat{f}\left(e_{m}\right)\right)$ where the edges of $G$ are indexed in such a way that $\widehat{f}\left(e_{i}\right) \leq \widehat{f}\left(e_{j}\right)$ for $i<j$, that is, in ascending order of their grey tones. For the sake of clarity and when the graph is fixed, it can be denoted $\operatorname{cont}(G, f)=\mathcal{C}_{f}$. Figure 2 shows two greyscales of the graph $K_{4}, f$ and $f^{\prime}$, whose corresponding contrast vectors are $\mathcal{C}_{f}=\left(0, \frac{1}{2}, \frac{1}{2}, \frac{1}{2}, \frac{1}{2}, 1\right)$ and $\mathcal{C}_{f^{\prime}}=\left(\frac{1}{3}, \frac{1}{3}, \frac{1}{3}, \frac{2}{3}, \frac{2}{3}, 1\right)$, respectively.

Given two greyscales $f$ and $f^{\prime}$ of a graph $G$, we say that $f$ has better contrast than $f^{\prime}$ if their corresponding contrast vectors verify $\mathcal{C}_{f}>\mathcal{C}_{f^{\prime}}$, following the lexicographical order. Thus, the ascending order of contrast vectors determines the goodness in terms of contrast. In Fig. 2, the greyscale $f^{\prime}$ has better contrast than $f$.

The maximum contrast vector is defined as

$$
\operatorname{cont}_{\text {max }}(G)=\max \{\operatorname{cont}(G, f) \text { such that } f \text { is a greyscale of } G\} \text {. }
$$

If $f$ is a greyscale of $G$ which gives rise to the vector $\operatorname{cont}_{\max }(G)$, we will say that $f$ is a maximum contrast greyscale of $G$ and the first component of $\operatorname{cont}_{\max }(G)$ will be called the lightest tone of $G$ and denoted $l t(G)$.

The following problem arises naturally in the context of contrast. It is posed for connected graphs, but general graphs can be also considered and analogous results hold when working with each one of their connected components.

\section{Maximum contrast on graphs (MACG)}

Instance: Connected graph $G(V, E)$.

Question: Is it possible to find a greyscale of $G$ such that its contrast vector is maximum?

We also deal with the restricted version of this problem, namely when the grey tones of some vertices are a priori known and the aim is to obtain the maximum contrast vector preserving these fixed grey tones.

Given a graph $G(V, E)$ and a nonempty proper subset $V_{c}$ of $V$, an incomplete $V_{c}$-greyscale of $G$ is a mapping of $V_{c}$ to the interval $[0,1]$. The vertices of $V_{c}$ are named initially coloured vertices. A greyscale $f$ is compatible with an incomplete $V_{c}$-greyscale $g$ if $f(u)=g(u)$ for all $u \in V_{c}$. The process of obtaining such an $f$ is 
called extending the incomplete greyscale $g$. We focus on a particular case, namely when only white and black tones are fixed. The problem is established as follows and it will be formally studied in Sect. 5 .

$\{0,1\}$-Restricted maximum contrast on graphs $(\{0,1\}$-RMACG)

Instance: Connected graph $G(V, E)$ and an incomplete $V_{c}$-greyscale $g$, where $V_{c}=$ $V_{0} \cup V_{1} \subset V$ with $V_{0}$ and $V_{1}$ disjoint subsets and such that $g(v)=0$ for $v \in V_{0}$ and $g(v)=1$ for $v \in V_{1}$.

Question: Is it possible to find a greyscale $f$ of $G$ compatible with $g$ such that its contrast vector is maximum in the set of contrast vectors of all possible greyscales compatible with $g$ defined on $G$ ?

If a greyscale $f$ provides an affirmative answer to the above problem we say that $f$ is a maximum contrast greyscale for the $\{0,1\}$-RMACG problem. Note that $f$ is a maximum contrast greyscale compatible with the greyscale given in the instance. The contrast vector associated to $f$ is named the maximum contrast vector for the $\{0,1\}$-RMACG problem.

\section{Maximum contrast problem}

The problem of finding out the maximum contrast of a connected graph, denoted MACG, is tackled in this section. Given a connected graph $G$, this problem consists of knowing whether a greyscale whose contrast vector is maximum can be found for $G$. A relation between the chromatic number and the lightest tone of $G$ is obtained. The main consequence of this property is the NP-completeness of the MACG problem. Next, the underlying structure of maximum contrast vectors is studied and some results related to it are established, which can be useful to develop approximation algorithms for the MACG problem.

The existence of the maximum element among the set of contrast vectors of a given graph will be guaranteed later, in Sect. 4 , due to the necessity of technical tools and specific results independently from the greyscale notion.

Nonetheless, according to the definition of better contrast, given in Sect. 2, it is clear that the maximum contrast vector has no component equal to 0 . Besides, it is immediately deduced that a necessary condition for a greyscale $f$ to be a maximum contrast greyscale of $G$ is that for any vertex $v$ with degree $1, f(v)$ is an extreme tone.

Next, the NP-completeness of the MACG problem is established. The proof of this result contains interesting relationships between greyscales and colourings of the given connected graph.

Theorem 1 The MACG problem is NP-complete.

Proof The key is to prove that $\chi(G)=1+\left\lceil\frac{1}{l t(G)}\right\rceil$, and since the chromatic number problem is NP-hard (Garey and Johnson 1979), the assertion holds.

For the sake of simplicity, let $k$ denote the natural number $\left\lceil\frac{1}{\operatorname{lt}(G)}\right\rceil$ and let $f$ be a maximum contrast greyscale of the graph $G$. The following mapping $\Phi: V \longrightarrow$ $\{0,1, \ldots, k\}$ is proven to be a $(k+1)$-colouring of $G$ : 


$$
\Phi(v)=\left\{\begin{array}{l}
i \text { if } \frac{i}{k} \leq f(v)<\frac{i+1}{k} \text { for } i=0, \ldots, k-1 \\
k \text { if } f(v)=1
\end{array}\right.
$$

On the contrary, suppose that there is an edge $e=\{u, v\} \in E$ such that $\Phi(u)=$ $\Phi(v)=i$ with $i \neq k$. Since $\frac{i}{k} \leq f(u)<\frac{i+1}{k}$ and $\frac{i}{k} \leq f(v)<\frac{i+1}{k}$ then $\widehat{f}(e)=1$ $f(u)-f(v) \mid<\frac{1}{k}=\frac{1}{\left\lceil\frac{1}{l t(G)}\right\rceil} \leq l t(G)$, which contradicts the definition of lightest tone. In addition, if $\Phi(u)=\Phi(v)=k$ then $\widehat{f}(e)=|f(u)-f(v)|=0$, contradicting that $\operatorname{cont}_{\max }(G)$ has no component equal to 0 .

The existence of the $(k+1)$-colouring $\Phi$ of $G$ ensures that $\chi(G) \leq k+1$. In order to prove the equality, let $\Psi: V \longrightarrow\{0,1, \ldots, r\}$ be a colouring of $G$ with $r+1=\chi(G)$ colours and we define $g: V \longrightarrow[0,1]$ a greyscale of $G$ as $g(v)=\frac{\Psi(v)}{r}$ for all $v \in V$. Hence,

$$
\widehat{g}(e)=|g(u)-g(v)|=\left|\frac{\Psi(u)}{r}-\frac{\Psi(v)}{r}\right|=\frac{1}{r}|\Psi(u)-\Psi(v)| \geq \frac{1}{r}
$$

for every $e=\{u, v\} \in E$. Then, $\operatorname{lt}(G) \geq \frac{1}{r}$, which implies that $\left\lceil\frac{1}{\operatorname{lt}(G)}\right\rceil \leq r$ and, since $r+1=\chi(G)$, we conclude that $\chi(G)=1+\left\lceil\frac{1}{\operatorname{lt}(G)}\right\rceil$.

The rest of this section is devoted to the study of the underlying structure of maximum contrast vectors and the establishment of their properties. Given the NPcompleteness nature of the MACG problem, the following results will be useful in order to design heuristic methods that provide high-quality solutions feasible in reasonable amounts of time.

The next technical lemma is needed to prove that $l t(G)=\frac{1}{\chi(G)-1}$, a more accurate relationship between $l t(G)$ and $\chi(G)$ than the one given in the proof of Theorem 1 , result that will be established in Theorem 2 .

Lemma 1 Let $G(V, E)$ be a connected graph and let $f$ be a maximum contrast greyscale of $G$. Let $v \in V$ be a vertex such that $0<f(v)<1$, then there exist $u_{1}$ and $u_{2} \in N(v)$ satisfying both of the following assertions:

1. $f\left(u_{1}\right)<f(v)<f\left(u_{2}\right)$.

2. $\widehat{f}\left(\left\{u_{1}, v\right\}\right)=\widehat{f}\left(\left\{u_{2}, v\right\}\right)=\min \{\widehat{f}(\{u, v\}): u \in N(v)\}$.

Proof Let us consider $a=\min \{\widehat{f}(\{u, v\}): u \in N(v)\}$. Since $f$ is a maximum contrast greyscale, then $a>0$ trivially and since $0<f(v)<1$, it is clear that $a<1$.

Let us define the set $\mathcal{A}=\{e \in E: \widehat{f}(e)=a\}$, then the maximum contrast vector has $|\mathcal{A}|$ coordinates equal to $a$, namely $\mathcal{C}_{f}=(\ldots, \underbrace{a, \ldots, a}, \ldots)$.

$|\mathcal{A}|$

Let us now partition the set $N(v)=\mathcal{B} \cup \mathcal{C}$ as follows:

$$
\mathcal{B}=\{u \in N(v): \widehat{f}(\{u, v\})=a\} \text { and } \mathcal{C}=\{u \in N(v): \widehat{f}(\{u, v\})>a\} .
$$


Let us define $b=\min \{\widehat{f}(\{u, v\}): u \in \mathcal{C}\}$ if $\mathcal{C} \neq \varnothing$ and $b=1$ otherwise. Since $a<b$, let us consider $\varepsilon>0$, such that $a+\varepsilon<b-\varepsilon, 0<f(v)-\varepsilon$ and $f(v)+\varepsilon<1$. Two new greyscales $f_{v^{+}}$and $f_{v^{-}}$are now defined as follows:

$$
f_{v^{+}}(w)=\left\{\begin{array}{ll}
f(w) & \text { if } w \neq v \\
f(v)+\varepsilon & \text { if } w=v
\end{array} \quad f_{v^{-}}(w)= \begin{cases}f(w) & \text { if } w \neq v \\
f(v)-\varepsilon & \text { if } w=v\end{cases}\right.
$$

By definition, the greyscales $f_{v^{ \pm}}$verify $\widehat{f}_{v^{ \pm}}\left(\left\{w_{1}, w_{2}\right\}\right)=\widehat{f}\left(\left\{w_{1}, w_{2}\right\}\right)$ for all edges $\left\{w_{1}, w_{2}\right\}$ of $G$ with $w_{1}, w_{2} \neq v$ and $\widehat{f_{v^{ \pm}}}\left(\left\{w_{1}, v\right\}\right)=a \pm \varepsilon<b \pm \varepsilon \leq \widehat{f}_{v^{ \pm}}\left(\left\{w_{2}, v\right\}\right)$ for all edges $\left\{w_{1}, v\right\},\left\{w_{2}, v\right\}$ of $G$ with $w_{1} \in \mathcal{B}$ and $w_{2} \in \mathcal{C}$.

Let us prove the statements by reductio ad absurdum. By assuming $f(u) \geq f(v)$ in the subset $\mathcal{B}$, we obtain that $\widehat{f}_{v^{-}}(\{u, v\})=a+\varepsilon$ for all $u \in \mathcal{B}$, and $\widehat{f}_{v^{-}}(\{u, v\})>a+\varepsilon$ for all $u \in \mathcal{C}$. In an analogous way, the assumption that $f(u) \leq f(v)$ leads to that $\widehat{f}_{v^{+}}(\{u, v\})=a+\varepsilon$ for all $u \in \mathcal{B}$, and $\widehat{f}_{v^{+}}(\{u, v\})>a+\varepsilon$ for all $u \in \mathcal{C}$. It is readily deduced that both contrast vectors associated to these greyscales only differ from $\mathcal{C}_{f}$ in the grey tones of the edges incident in $v$, and contain $|\mathcal{A}|-|\mathcal{B}|$ components equal to $a$ and at least $|\mathcal{B}|$ components equal to $a+\varepsilon$ :

$$
\mathcal{C}_{f_{v^{ \pm}}}=(\ldots, \underbrace{a, \ldots, a}_{|\mathcal{A}|-|\mathcal{B}|}, \underbrace{a+\varepsilon, \ldots, a+\varepsilon}_{\geq|\mathcal{B}|}, \ldots) .
$$

In addition, it is straightforward to verify that $\mathcal{C}_{f}$ and $\mathcal{C}_{f_{v^{ \pm}}}$both have exactly the same components smaller than $a$.

Otherwise, $\mathcal{C}_{f_{v^{ \pm}}}$can contain values not belonging to $\mathcal{C}_{f}$, but they are necessarily greater than $a+\varepsilon$, as a matter of fact, greater than $b-\varepsilon$.

Obviously, the contrast vectors $\mathcal{C}_{f_{v^{ \pm}}}$are better than $\mathcal{C}_{f}$, contradicting that $f$ is a maximum contrast greyscale. Consequently, both assumptions $f(v) \leq f(u)$ and $f(u) \leq f(v)$ for all $u \in \mathcal{B}$ are false and there is at least one vertex $u_{1} \in \mathcal{B}$ and one vertex $u_{2} \in \mathcal{B}$ such that $f\left(u_{1}\right)<f(v)<f\left(u_{2}\right)$ and $\widehat{f}\left(\left\{u_{1}, v\right\}\right)=\widehat{f}\left(\left\{u_{2}, v\right\}\right)=a$.

The pair of vertices $u_{1}$ and $u_{2}$ associated to a vertex $v$, given by Lemma 1 , will be named pair of neighbours closest to $v$, the vertex $u_{1}$ will be named the neighbour closest to $v$ on the left and the vertex $u_{2}$ will be named the neighbour closest to $v$ on the right.

Let us remark that the existence of such pair of vertices $u_{1}$ and $u_{2}$, together with the below-defined notion of incremental path, are the keys to know the underlying structure of maximum contrast vectors. The study of $\operatorname{Im}(f)$, for any maximum contrast greyscale $f$ of $G$, will be addressed in detail in Sect. 4 and, again, the pair of neighbours closest to a vertex will be determinant in it.

Given a greyscale $f$ of $G$, an incremental path of length $k \in N$ for $f$ is defined to be a path of $G, P_{k}=\left\{u_{0}, e_{1}, u_{1}, e_{2}, u_{2}, \ldots, e_{k}, u_{k}\right\}$ with $f\left(u_{i}\right)=\frac{i}{k}$ for $i=0,1, \ldots, k$. Thus, in any incremental path of length $k$ all edges are coloured with the grey tone 
$\frac{1}{k}$. We show that if $f$ is a maximum contrast greyscale of $G$, then the lightest tone is placed in edges defining some incremental paths.

Theorem 2 Let $G(V, E)$ be a connected graph with $\chi(G)=k+1$ and let $f$ be a maximum contrast greyscale of $G$ with lightest tone $l t(G)$. The following statements hold:

1. $\operatorname{lt}(G)=\frac{1}{k}$.

2. For everye $\in E$ with $\widehat{f}(e)=\operatorname{lt}(G)$ there is at least one incremental path of length $k$ containing $e$.

3. The vector cont $t_{\text {max }}(G)$ has at least $k$ components equal to $l t(G)$.

4. The set $I_{k}=\left\{\frac{i}{k}: i=0,1, \ldots, k\right\}$ is a subset of $\operatorname{Im}(f)$.

Proof On one hand, the case of $l t(G)=1$ leads to the maximum contrast vector whose all components are equal to 1 , and so $\operatorname{Im}(f)=\{0,1\}$, that is, $G$ is a 2-chromatic connected graph and $k=1$. Then, given an edge $e \in E$ with $\widehat{f}(e)=\operatorname{lt}(G)=1$, the incremental path of length 1 is precisely the edge $e$.

On the other hand, let us consider an edge $e=\left\{u_{0}, v_{0}\right\}$ such that $\widehat{f}(e)=\operatorname{lt}(G)<1$. In this case, it is clear that $\left\{f\left(u_{0}\right), f\left(v_{0}\right)\right\} \neq\{0,1\}$ and at least one of these vertices has degree at least two since $G$ is connected. Without loss of generality, we suppose that $f\left(u_{0}\right)<f\left(v_{0}\right)$.

Firstly, let us consider the case $0<f\left(u_{0}\right)<f\left(v_{0}\right)<1$. Lemma 1 applied to $v_{0}$ ensures the existence of a neighbour $v_{1}$ closest to $v_{0}$ on the right satisfying $\widehat{f}\left(\left\{v_{0}, v_{1}\right\}\right)=\operatorname{lt}(G)$. Again, by applying Lemma 1 to the vertex $v_{1}$, it is possible to obtain a new neighbour $v_{2}$ closest to $v_{1}$ on the right such that $\widehat{f}\left(\left\{v_{1}, v_{2}\right\}\right)=\operatorname{lt}(G)$ and by iterating this process, a sequence of neighbours closest on the right $\left\{v_{0}, v_{1}, \ldots, v_{s}\right\}$ such that $f\left(v_{s}\right)=1$ and $s=\left[\frac{1-f\left(v_{0}\right)}{l t(G)}\right]$ is constructed. Analogous reasoning is applied to the left of $u_{0}$. In this situation, Lemma 1 ensures the existence of a neighbour $u_{1}$ closest to $u_{0}$ on the left. Again, by iterating this procedure, it is possible to obtain a sequence of neighbours closest on the left $\left\{u_{r}, u_{r-1}, \ldots, u_{0}\right\}$ such that $f\left(u_{r}\right)=0$ and $r=\left[\frac{f\left(u_{o}\right)}{l t(G)}\right]$.

Let $P_{t}=\left\{u_{r}, \ldots, u_{0}, v_{0}, v_{1}, \ldots, v_{s}\right\}$, with $t=r+s+1$, stand for the incremental path for $f$. Note that $P_{t}$ satisfies $0=f\left(u_{r}\right)<f\left(u_{i-1}\right)<\cdots<f\left(u_{0}\right)<f\left(v_{0}\right)<$ $\ldots f\left(v_{s}\right)=1$.

Secondly, the case of $0=f\left(u_{0}\right)<f\left(v_{0}\right)<1$ leads to a path $P_{t}$ starting with $u_{0}$, that is $r=0$. Analogously, in case that $0<f\left(u_{0}\right)<f\left(v_{0}\right)=1$ the path $P_{t}$ finishes with $v_{0}$, that is $s=0$.

Finally, from the above construction, the interval $[0,1]$ is divided into $t$ subintervals of equal length $l t(G)$, hence $l t(G)=\frac{1}{t}$ with $t$ a natural number. Since $\chi(G)=k+1$ and according to the proof of the NP-completeness of the MACG problem (Theorem 1), it holds that $\chi(G)=k+1=1+\left\lceil\frac{1}{\operatorname{lt}(G)}\right\rceil=1+t$, and so $t=k$. Thus, $\operatorname{lt}(G)=\frac{1}{k}$ and assertions 1 and 2 follow. Observe that the grey tone of the $i$-th vertex of $P_{k}$ is $\frac{i}{k}$ (for $i=0,1, \ldots, k$ ) and consequently all its edges have grey tone equal to $l t(G)$. Therefore, statements 3 and 4 hold and the proof is finished. 
The lightest tone of a given connected graph is a new invariant which has been characterized through its relationship with the chromatic number of the graph. This fact has directly led to the NP-completeness of the MACG problem. Furthermore, some properties have been established in the context of maximum contrast, in particular the characterization of the first components of maximum contrast vectors. In the next section, we will study all possible values of any maximum contrast greyscale and we will prove that these numbers are evenly distributed along particular paths in a similar way as the greytones of the edges in maximum contrast vectors along incremental paths.

\section{Characterizing the maximum contrast greyscales}

We can set two objectives in this section. Firstly, even if the MACG problem is defined in Sect. 2 and its NP-completeness is demonstrated in Sect. 3, the current problem would not be well defined until we prove that for any graph $G$, the set $\{\operatorname{cont}(G, f)$ : $f$ is a greyscale of $G\}$ has a maximum, that is, the existence of the maximum contrast vector $\operatorname{cont}_{\max }(G)$ for any graph $G$. This way, it suffices to show that $\operatorname{Im}(f)$ is a finite set for any graph $G(V, E)$. Thus, Sect. 4 is about the main objective of proving that the set $\operatorname{Im}(f)$ is finite.

Secondly, Theorem 2 proves that the lightest tone $l t(G)$ is assigned to the edges of an incremental path, this is, a path among the graph $G$ with all its edges coloured with a $l t(G)$ greytone and extreme vertices having a greytone 0 and 1 . Similarly, from the existence of pair of neighbours closest to a vertex and statement 2 of Theorem 2, we observe that for any vertex $v$ with colour other than the extreme tones, there exists a path of length at least 2 verifying that $v$ is an interior point of that path. Moreover, the vertices of such path are coloured with an increasing sequence of grey tones and all edges have the same grey tone. We may guess that $\operatorname{Im}(f)$ consists of the union of some sets of values corresponding to the colours of such paths and there are certain relations between those sets.

Consequently, both objectives set out in this section lead us to study the characterization of maximum contrast greyscales by means of studying the underlying structure in set $\operatorname{Im}(f)$. We study this fact in a deeper way as follows.

In accordance with Theorem 2 , for any connected graph $G(V, E)$, it is known that the set $I_{k}=\left\{\frac{i}{k}: i=0,1, \ldots, k\right\}$ with $k=\frac{1}{l t(G)}$ is a subset of $\operatorname{Im}(f)$. It is not difficult to find a graph for which the maximum contrast greyscale $f$ verifies $I_{k} \subsetneq \operatorname{Im}(f)$. In Fig. 1 (top left) a maximum contrast greyscale of the wheel is given: $f([0,1,2,3,4,5])=\left[1,0, \frac{1}{2}, 0, \frac{2}{3}, \frac{1}{3}\right]$, hence $I_{3} \subsetneq \operatorname{Im}(f)$.

Now, we introduce some notions and notation that will be usefull in order to describe how $\operatorname{Im}(f)$ is structured. A sequence $\left[y_{0}, y_{1}, \ldots, y_{r}\right] \subset[0,1]$, with $r \geq 2$, is said to be an $h$-step chain of length $r$ in $[0,1]$ if $y_{i}-y_{i-1}=h$ for $i=1, \ldots, r$. A number $y_{i}$ for $i \neq 0, r$ is named interior point and $y_{0}$ and $y_{r}$ are called extreme points of the $h$-step chain. Let us observe that any $h$-step chain is characterized by its extreme points $y_{0}$ and $y_{r}$ and its length $r$, being $h=\frac{y_{r}-y_{0}}{r}$.

A set of numbers $F \subset[0,1]$ is said to be an h-minimum-step-enchained set if $F$ verifies the following assertions: 
(1) There is an $h$-step chain in $F$ whose extreme points are precisely $\{0,1\}$ and there is no other $q$-step chain in $F$ with extreme points $\{0,1\}$ and $q<h$.

(2) For every $y \in F-\{0,1\}$ there exists a $p$-step chain $P$ in $F$ with extreme points $y_{1}$ and $y_{2}$ and such that $y$ is an interior point in $P$ for some $p \geq h$.

If $p>h$ and $y_{i} \notin\{0,1\}$, for $i=1$ or 2 , then $y_{i}$ is an interior point of a $q_{i}$-step chain with $h \leq q_{i}<p$.

An $h$-minimum-step-enchained set $F$ is maximal if it is not a proper subset of another $h$-minimum-step-enchained set.

Let us illustrate these notions with an example. $F=\left\{0, \frac{1}{4}, \frac{3}{8}, \frac{1}{2}, \frac{5}{8}, \frac{3}{4}, 1\right\}$ is a $\frac{1}{4}$ minimum-step-enchained set containing the $\frac{1}{4}$-step chain $\left[0, \frac{1}{4}, \frac{1}{2}, \frac{3}{4}, 1\right]$, the $\frac{3}{8}$-step chains $\left[0, \frac{3}{8}, \frac{3}{4}\right]$ and $\left[\frac{1}{4}, \frac{5}{8}, 1\right]$, and the $\frac{1}{2}$-step chain $\left[0, \frac{1}{2}, 1\right]$. $F$ also contains the $\frac{1}{8}$-step chain $\left[\frac{1}{4}, \frac{3}{8}, \frac{1}{2}, \frac{5}{8}, \frac{3}{4}\right]$, which does not contradict the definition of $\frac{1}{4}$-minimumstep-enchained set.

The next theorem gives a characterization of any maximum contrast greyscale $f$ of a given graph $G$, by describing the underlying structure of the set $\operatorname{Im}(f)$.

Theorem 3 Let $G(V, E)$ be a connected graph with chromatic number $\chi(G)=k+1$. For any maximum contrast greyscale $f$ of $G, \operatorname{Im}(f)$ is a $\frac{1}{k}$-minimum-step-enchained set.

Proof Let us consider an arbitrary maximum contrast greyscale $f$ of $G$. Firstly, we show that $\operatorname{Im}(f)$ satisfies assertion (1) in the definition of $\frac{1}{k}$-minimum-step-enchained set. From Theorem 2 it holds that $I_{k} \subset \operatorname{Im}(f)$ is a $\frac{1}{k}$-step chain with extreme points $\{0,1\}$, where $k=\chi(G)-1$. Suppose there exists a $q$-step chain with extreme points $\{0,1\}$ and $q<\frac{1}{k}$, namely $[0, q, \ldots, 1]$. Then, $q=f(v)$ for some $v \in V$ and, by Lemma 1, there exists $u_{1} \in N(v)$, a neighbour closest to $v$ on the left. Hence, $f\left(u_{1}\right)<$ $f(v)$ and $\widehat{f}\left(\left\{u_{1}, v\right\}\right) \leq q<\frac{1}{k}$, the lightest tone, which is a contradiction. Therefore, $I_{k}$ is the only $\frac{1}{k}$-chain with extremes $\{0,1\}$ and assertion (1) holds.

In order to prove assertion (2) in the definition of $\frac{1}{k}$-minimum-step-enchained set we use the following auxiliary mapping $C: V \longrightarrow[0,1]$ defined as follows:

$$
C(v)=\min _{w \in f^{-1}(f(v))}\{\widehat{f}(\{u, w\}): u \in N(w)\}
$$

In other words, $C$ computes the minimum grey tone over the set of all edges incident in vertices coloured with the grey tone $f(v)$.

Given a value $y \in \operatorname{Im}(f)-\{0,1\}$, let $v$ be a vertex such that $y=f(v)$. Let us consider $C(v)$ and a vertex $w \in C^{-1}(C(v))$. Since $f(w)=y \notin\{0,1\}$, the hypotheses of Lemma 1 are verified and hence there is a pair of neighbours closest to $w$, namely $u_{1}$ and $v_{1}$ such that $f\left(u_{1}\right)<f(w)<f\left(v_{1}\right)$ and $\widehat{f}\left(\left\{u_{1}, w\right\}\right)=\widehat{f}\left(\left\{v_{1}, w\right\}\right)=p$, where $p=C(v)$. Therefore, $\left[f\left(u_{1}\right), f(w)=y, f\left(v_{1}\right)\right]$ is a $p$-step chain. Moreover, and since $w \in N\left(u_{1}\right)$, it is held that $C\left(u_{1}\right) \leq \widehat{f}\left(\left\{u_{1}, w\right\}\right)=C(w)=C(v)=p$ (see Lemma 1); analogously $C\left(v_{1}\right) \leq p$.

Let us suppose $C\left(u_{1}\right)=C(w)=p$ and $f\left(u_{1}\right) \neq 0$. A similar reasoning gives rise to $w_{2} \in C^{-1}\left(C\left(u_{1}\right)\right)$ and there exists $u_{2}$, the neighbour closest to $w_{2}$ on the left such that $f\left(u_{2}\right)=f\left(u_{1}\right)-p$, and the above $p$-step chain is enlarged on the 
left as follows $\left[f\left(u_{2}\right), f\left(u_{1}\right), f(w)=y, f\left(v_{1}\right)\right]$. This procedure can be repeated $r_{1}$ times until $C\left(u_{r_{1}}\right)<C(w)=p$ or else $f\left(u_{r_{1}}\right)=0$. This way, a left extreme point for the $p$-step chain with interior point $y$ is found. In summary, we obtain $\left[f\left(u_{r_{1}}\right), \ldots, f\left(u_{2}\right), f\left(u_{1}\right), f(w)=y, f\left(v_{1}\right)\right]$. The case $C\left(v_{1}\right)=C(w)=p$ and $f\left(v_{1}\right) \neq 1$ can be tackled similarly and the $p$-step chain is completed on the right in the form: $\left[f\left(u_{r_{1}}\right), \ldots, f\left(u_{2}\right), f\left(u_{1}\right), f(w)=y, f\left(v_{1}\right), f\left(v_{2}\right), \ldots, f\left(v_{r_{2}}\right)\right]$ whose extreme points are 0 or 1 or otherwise $C$ reaches a value less than $p$.

Notice that, from the definition of lightest tone of $G$, for every vertex $u, C(u) \geq \frac{1}{k}$ holds, in particular $C(v)=p \geq \frac{1}{k}$. Therefore, every $y \in \operatorname{Im}(f)-\{0,1\}$ is an interior point of a $p$-step chain with $p \geq \frac{1}{k}$ and whose extreme points are $f\left(u_{r_{1}}\right)=0$ or else $C\left(f\left(u_{r_{1}}\right)\right)=q_{1}<p$ or $f\left(v_{r_{2}}\right)=1$ or else $C\left(f\left(v_{r_{2}}\right)\right)=q_{2}<p$. In such a case, a similar construction gives rise to a $q_{i}$-step chain with $q_{i}<p$ and $f\left(u_{r_{1}}\right)$ or $f\left(v_{r_{2}}\right)$ are the corresponding interior points. We conclude that $\operatorname{Im}(f)$ is a $\frac{1}{k}$-minimum-stepenchained set.

Theorem 5 shows that for any fixed natural number $k \geq 2$, there exits a unique maximal $\frac{1}{k}$-minimum-step-enchained set, denoted $F_{k}$ in the following, which is a finite set. We will postpone the proof of this relevant fact for ease of reading of this section. Therefore, as an immediate consequence of the finiteness of $F_{k}$ we reach the next main result assuring our first goal, that is the notion of maximum contrast vector is well defined.

Theorem 4 For any graph $G$ with chromatic number $\chi(G)=k+1$, the set $\{\operatorname{cont}(G, f): f$ is a greyscale of $G\}$ has a maximum. Furthermore, for any maximum contrast greyscale $f$ of $G, I_{k} \subset \operatorname{Im}(f) \subset F_{k}$ is verified.

Next, let us focus on the study of $F_{k}$. We design a recursive procedure that gives a maximal $\frac{1}{k}$-minimum-step-enchained set for each $k \geq 2$. Later we will prove that it is unique $y$ finite. The procedure starts with the set $\{0,1\}$ and adds all possible values $y \in(0,1)$ that guarantee the definition of $\frac{1}{k}$-minimum-step-enchained set is verified. More particularly, the method adds all possible $p$-step chains, with $p \geq \frac{1}{k}$, in such a way that its interior and extreme points verify assertion (2) of the definition for minimum step equal to $\frac{1}{k}$.

We define the auxiliary mapping $S_{H, k}$ which will help us checking assertion (2) of the definition of minimum-step-enchained set, during the procedure given below.

Let $H \subset[0,1]$ be a finite set of numbers and $k \in N$ with $k \geq 2$. The function $S_{H, k}: H \longrightarrow[0,1]$ is defined by:

$$
S_{H, k}(y)=\left\{\begin{array}{c}
\min \left\{p \geq \frac{1}{k} \quad: y \text { is an interior point for some } p-\text { step chain in } H\right. \\
\quad \text { verifying assertion (2) of minimum-step-enchained set }\} \\
0 \text { otherwise }
\end{array}\right.
$$

The following procedure starts with the set $H=\{0,1\}$ and adds possible values of new $p$-step chains $\left[y_{0}, \ldots, y_{r}\right]$ with $p \geq \frac{1}{k}$ recursively whenever $\max \left\{S_{H, k}\left(y_{0}\right), S_{H, k}\left(y_{r}\right)\right\}<p$ is true. These new values are stored in the set $H$ and $S_{H, k}$ must be updated in each step. The algorithm ends when it is not possible 
to add a new interior point of a chain that satisfies assertion (2) of the definition of $h$-minimum-step-enchained set.

PROCEDURE: MAXIMAL ENCHAINED SET (MES)

Input: A natural number $k \geq 2$.

Output: The maximal $\frac{1}{k}$-minimum-step-enchained set, $F_{k}$.

1. Initialize $H, N e w \leftarrow\{0,1\}$

2. Initialize $S_{H, k}(y)=0$ for all $y \in H$

3. While $|N e w|>0$ do

(a) Initialize $N e w \leftarrow \emptyset$

(b) For each $\left\{y_{1}, y_{2}\right\} \subset H$ do

i. Initialize $r \leftarrow 2$

ii. Initialize $p \leftarrow \frac{\left|y_{2}-y_{1}\right|}{r}$

iii. While $p \geq \frac{1}{k}$ do

A. If $p>\max \left\{S_{H, k}\left(y_{1}\right), S_{H, k}\left(y_{2}\right)\right\}$ do

I. Make $C_{r}$ the $p$-step chain with extremes $\left\{y_{1}, y_{2}\right\}$.

II. For each $y \in C_{r}-\left\{y_{1}, y_{2}\right\}$ do

$\alpha$. If $y \notin H$ then $S_{H, k}(y)=p$

$\beta$. If $S_{H, k}(y)>p$ then $S_{H, k}(y)=p$

III. Update New $\leftarrow\left(\right.$ New $\left.\cup\left(C_{r}-H\right)\right)$

B. Update $r \leftarrow r+1$

C. Update $p \leftarrow \frac{\left|y_{2}-y_{1}\right|}{r}$

(c) Update $H \leftarrow(H \cup N e w)$

4. return $F_{k}=H$

Since the procedure starts with two fixed numbers, these are $\{0,1\}$, and each step is deterministic, the uniqueness of its output is straightforwardly deduced. Notice that this affirmation is true whenever the finiteness of the procedure is proven (Theorem 5). By construction, the updated $H$ after the execution of Step 3.(c) is a $\frac{1}{k}$-minimum-stepenchained set. The output of the algorithm is the maximal $\frac{1}{k}$-minimum-step-enchained set, $F_{k}$, due to the fact that every pair of possible values for $H$ are revisited in Step 3.(b) until no new value can be adjoined to $H$. Moreover, $F_{k}$ is a rational set since only rational numbers are adjoined to $C_{r}$ in Step 3.(b)iii.A.I of the algorithm.

Surprisingly, although the MACG problem is defined over the real interval $[0,1]$, its solution is obtained by subdividing $[0,1]$ in equal parts a finite number of times recursively. This way, the maximum contrast greyscale has all values rational.

We have run the MES procedure for $k$ from 2 to 7 . Knowing such sets $F_{k}$ will allow us to obtain some examples of maximum contrast of small graphs. The following sets are obtained:

$F_{2}=\left\{0, \frac{1}{2}, 1\right\}, F_{3}=\left\{0, \frac{1}{3}, \frac{1}{2}, \frac{2}{3}, 1\right\}, F_{4}=\left\{0 \frac{1}{4}, \frac{1}{3}, \frac{3}{8}, \frac{1}{2}, \frac{5}{8}, \frac{2}{3}, \frac{3}{4}, 1\right\}$,

$F_{5}=\left\{0, \frac{1}{5}, \frac{1}{4}, \frac{4}{15}, \frac{3}{10}, \frac{1}{3}, \frac{7}{20}, \frac{11}{30}, \frac{3}{8}, \frac{2}{5}, \frac{7}{15}, \frac{19}{40}, \frac{1}{2}, \frac{21}{40}, \frac{8}{15}, \frac{3}{5}, \frac{5}{8}, \frac{19}{30}, \frac{13}{20}, \frac{2}{3}, \frac{7}{10}, \frac{11}{15}, \frac{3}{4}\right.$, $\left.\frac{4}{5}, 1\right\}$,

$F_{6}=\left\{0, \frac{1}{6}, \frac{1}{5}, \frac{5}{24}, \frac{2}{9}, \frac{1}{4}, \frac{7}{27}, \frac{19}{72}, \frac{4}{15}, \ldots\right\}, F_{7}=\left\{0, \frac{1}{7}, \frac{1}{6}, \frac{6}{35}, \frac{5}{28}, \frac{4}{21}, \frac{1}{5}, \frac{17}{84}, \frac{23}{112}, \ldots\right\}$. 
Table 1 Cardinality of some $F_{k}$

\begin{tabular}{lllllll}
\hline $\mathrm{k}$ & 2 & 3 & 4 & 5 & 6 & 7 \\
\hline$\left|F_{k}\right|$ & 3 & 5 & 9 & 25 & 145 & 19027 \\
\hline
\end{tabular}

The sets $F_{6}$ ad $F_{7}$ are collected in a data file which is available at de Castro et al. (2016). Table 1 shows the increase of $F_{k}$ cardinality. Note that the cases for $k \geq 6$ are particularly significant.

The finiteness of $F_{k}$ (and consequently of the MES procedure) deserves a detailed analysis, which starts with the following technical lemma. Let us establish some notation.

Fixed a natural number $k \geq 2$, we define a sequence of sets denoted $\left\{A_{i}\right\}$ for $i \geq 0$ by recurrence:

$A_{0}=\{0,1\}$

$A_{i}=\{$ interior points of p-chains verifying assertion (2) and such that its extremes belong to $\left.\cup_{j=0}^{i-1} A_{j}\right\}$

This way, the set $F_{k}$ is equivalent to $F_{k}=\cup_{i=0}^{\infty} A_{i}$. For the sake of simplicity, from now on we will denote $F_{k}^{i}=\cup_{j=0}^{i} A_{j}$. Let us illustrate these definitions with an example, for $k=4$, it is revealed $F_{4}=\cup_{i=0}^{\infty} A_{i}$, where $A_{0}=\{0,1\}, A_{1}=$ $\left\{\frac{1}{4}, \frac{1}{3}, \frac{1}{2}, \frac{2}{3}, \frac{3}{4}\right\}, A_{2}=\left\{\frac{3}{8}, \frac{5}{8}\right\}$, and $A_{j}=\emptyset$ for $j \geq 3$, that is, $F_{4}=F_{4}^{2}$.

Lemma 2 The following statements hold for any $A_{i}$ with $i \geq 1$ :

1. Every number $y \in A_{i}$ is an interior point for a p-step chain in $F_{k}$ with extreme points $y_{1}<y_{2}$ and $\left\{y_{1}, y_{2}\right\} \cap A_{i-1} \neq \emptyset$, where $p=S_{F_{k}^{i}, k}(y)$.

2. Symetry property: if $y \in A_{i}$ then $1-y \in A_{i}$ and $S_{F_{k}^{i}, k}(y)=S_{F_{k}^{i}, k}(1-y)$.

3. $A_{i}$ is a finite set.

4. Let be $p_{i}=\min \left\{S_{F_{k}^{i}, k}(y),: y \in A_{i}\right\}$, then $p_{i}>p_{i-1}$, where $p_{0}=0$.

Proof Statement 1 holds by definition of the set $A_{i}$. Statement 2 is demonstrated by induction over $i$ : it is trivially true for $A_{0}=\{0,1\}$ and let us suppose that it is also true for $A_{j}$ with $j \leq i$. Let us consider $y \in A_{i+1}$. Then, $y$ is an interior point of a $p$-step chain with extreme points $y_{1}<y_{2}$, such that $y_{s} \in F_{k}^{i}$ and $S_{F_{k}^{i+1}, k}(y)>\max \left\{\left(S_{F_{k}^{i}, k}\left(y_{1}\right), S_{F_{k}^{i}, k}\left(y_{2}\right)\right\}\right.$ for $s=1,2$. From the induction hypothesis, $1-y_{s} \in F_{k}^{i}$ and $S_{F_{k}^{i}, k}\left(1-y_{s}\right)=S_{F_{k}^{i}, k}\left(y_{s}\right)$, for $s=1$, 2. We make the $p$-step chain with extreme points $1-y_{2}<1-y_{1}$. Then, $1-y$ is an interior point and $S_{F_{k}^{i+1}, k}(1-y)=$ $S_{F_{k}^{i+1}, k}(y)>\max \left\{S_{F_{k}^{i}, k}\left(y_{1}\right), S_{F_{k}^{i}, k}\left(y_{2}\right)\right\}=\max \left\{S_{F_{k}^{i}, k}\left(1-y_{1}\right), S_{F_{k}^{i}, k}\left(1-y_{2}\right)\right\}$, therefore $1-y \in A_{i+1}$.

Statement 3 is demonstrated by induction over $i$ : it is true for $i=0$, since $\left|A_{0}\right|=2$. Let us suppose $\left|A_{j}\right|<+\infty$ for $j \leq i$, then $\left|F_{k}^{i}\right|=\left|\cup_{j=0}^{i} A_{j}\right| \leq$ $\left|A_{0}\right|+\left|A_{1}\right|+\cdots+\left|A_{i}\right|<+\infty$. According to Step 3 (b) iii.A.I. of MES procedure, in $A_{i+1}$ there are at most $\left(\begin{array}{c}\left|F_{k}^{i}\right| \\ 2\end{array}\right)$ chains with length $2,3, \ldots k$. Therefore, $\left|A_{i+1}\right|$ has at most

$$
(1+2+\cdots+(k-1))\left(\begin{array}{c}
\left|F_{k}^{i}\right| \\
2
\end{array}\right)=\frac{(k)(k-1)}{2}\left(\begin{array}{c}
\left|F_{k}^{i}\right| \\
2
\end{array}\right)
$$


elements, that is, $\left|A_{i+1}\right|<+\infty$.

For the proof of statement 4 , let us observe that if $y \in A_{i}$ then there is some $h$-step chain with extreme points $y_{1}<y_{2}$ and

$$
h=S_{F_{k}^{i}, k}(y)>\max \left\{S_{F_{k}^{i-1}, k}\left(y_{1}\right), S_{F_{k}^{i-1}, k}\left(y_{2}\right)\right\}
$$

and from statement $2, y_{1}$ or $y_{2}$ belongs to $A_{i-1}$, hence $S_{F_{k}^{i-1}, k}\left(y_{s}\right) \geq p_{i-1}$ for some $s=1,2$. Then,

$$
S_{F_{k}^{i}, k}(y)>\max \left\{S_{F_{k}^{i-1}, k}\left(y_{1}\right), S_{F_{k}^{i-1}, k}\left(y_{2}\right)\right\} \geq p_{i-1},
$$

that is, $S_{F_{k}^{i}, k}(y)>p_{i-1}$ for each $y \in A_{i}$. Since $\left|A_{i}\right|<+\infty$, it must be $p_{i}=$ $\min \left\{S_{F_{k}^{i}, k}(y)\right.$, such that $\left.y \in A_{i}\right\}>p_{i-1}$.

Finally, we are ready to demonstrate the announced theorem of finiteness of the set $F_{k}$.

Theorem $5 F_{k}$ is a finite set for every $k \geq 2$.

Proof By reductio ad absurdum, let us suppose $\left|F_{k}\right|=+\infty$. Since $F_{k}=\cup_{i=0}^{\infty} A_{i}$ and each set $A_{i}$ is finite, then it must be $A_{i} \neq \emptyset$ for all $i$, and hence, $\left\{p_{i}\right\}_{i=0}^{+\infty}$ is an infinite strictly increasing succession of numbers with trivial upper bound $p_{i}<\frac{1}{2}$. Then, there exists $\lim _{i \rightarrow+\infty} p_{i}=p$.

Otherwise, for each $a \in A_{i}$ and $0<a<\frac{1}{3}$, by the symmetry property, the number $b=1-a>\frac{2}{3}$ lies in $A_{i}$ and $c=\frac{b}{2}=\frac{1-a}{2} \in A_{i+1}$ because $c$ is an interior point of the $c$-step chain $[0, c, b]$ with $S_{F_{k}, k}(c)=c>\frac{1}{3}>S_{F_{k}, k}(b)=S_{F_{k}, k}(a)$. Let us observe that $\left(c-\frac{1}{3}\right)=\frac{1-a}{2}-\frac{1}{3}=\frac{\frac{1}{3}-a}{2}$, that is, for every $a \in A_{i}$ with $a<\frac{1}{3}$ there exists $c \in A_{i+1}$ with $\left|c-\frac{1}{3}\right|=\frac{1}{2}\left|a-\frac{1}{3}\right|$. Under the assumption $F_{k}$ is an infinite set, then value $\frac{1}{3}$ is an accumulation point in $F_{k}$ and then, $p=\lim _{i \rightarrow+\infty} p_{i}=\frac{1}{3}$.

Now, by definition of limit, for all $\epsilon>0$, there is some $m$ such that if $i \geq m$ then $\frac{1}{3}-p_{i}<\epsilon$. Let be $y \in A_{m+1}$, then $y$ is an interior point of an $h$-step chain with extreme points $y_{1}<y_{2}$ and some $y_{s} \in A_{m}$ for $s=1,2$, where $h \geq p_{m+1}$. Let us suppose $y_{2} \in A_{m}$, then $y_{2}$ is an interior point of a $q$-step chain with extreme points $y_{3}<y_{4}$, where $q \geq p_{m}$. Then $0<y_{1}<y<y_{2}<y_{4}<1$ and $y_{4}-y_{1}=$ $\left(y_{4}-y_{2}\right)+\left(y_{2}-y\right)+\left(y-y_{1}\right) \geq p_{m+1}+p_{m+1}+p_{m}>3\left(\frac{1}{3}-\epsilon\right)=1-3 \epsilon$. recall that $y_{4}-y_{1} \geq 1-\frac{1}{k}$, and then, $1-3 \epsilon<1-\frac{1}{k}$, so $\epsilon>\frac{1}{3 k}$ which is a contradiction. Therefore, the assumption is false and the set $F_{k}$ is finite for every $k \geq 2$.

As a consequence of Theorem 5, if $H_{i}$ denotes the set $H$ after $i$ loops in MES procedure, then the number of loops is finite because $F_{k}$ is finite. This demonstrates the finiteness of the procedure. Otherwise, from the proof of statement 3 in Lemma 2, let us remark that $H_{i}$ can be computed in $\mathcal{O}\left(\left|H_{i}\right|\right)$ time complexity. The complexity would be exponential, independently of the value of $k$ (and such a complexity would make the method impractical for large values of $k$ ). 
Fig. 3 A maximum contrast greyscale of the graph $G$ with $\chi(G)=5$ and $\operatorname{Im}(f)=F_{4}$

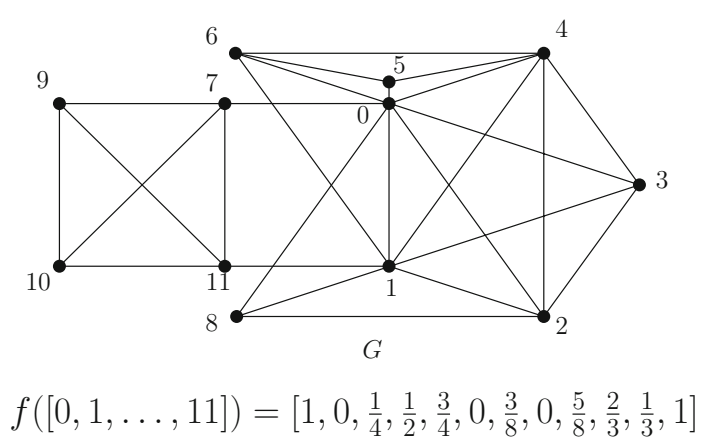

A natural question arises: we wonder if the bound set $\operatorname{Im}(f) \subset F_{k}$ for a given maximum contrast greyscale $f$ is tight or otherwise there exists $y \in F_{k}$ such that no graph $G(V, E)$ with $\chi(G)=k+1$ and no maximum contrast greyscale $f$ of $G$ verifies $y \in \operatorname{Im}(f)$. For $k=2$ and the complete graph $G=K_{3}, \operatorname{Im}(f)=F_{2}$. Figures $1(($ a) top) and 3 show two graphs for which $\operatorname{Im}(f)=F_{k}$, with $k=3,4$, respectively. A brute-force algorithm has been implemented for these two graphs in order to check that a maximum contrast greyscale has precisely these sets of values. Let us remark that in the latter case, the computation for $k=4$ requires at most $\left|F_{4}\right|^{n}=12^{9}$ combinations of values to test maximum contrast greyscales. In the general case, we guess that the bound $\operatorname{Im}(f) \subset F_{k}$ is tight but constructing particular examples verifying it is a difficult task since an amount of vertices $|V| \geq\left|F_{k}\right|$ is required and we are dealing with an NP-complete problem.

\section{Restricted maximum contrast problem on bipartite graphs}

In this section we tackle the restricted version of the maximum contrast problem in which the graph has a set $V_{c}$ of vertices initially coloured with extreme tones 0 (white) and 1 (black) and the remaining vertices must be coloured by preserving those initial colours. It is defined as $\{0,1\}$-Restricted maximum contrast on graphs $(\{0,1\}$ RMACG) in Sect. 2.

In the previous section, the NP-completeness of the MACG problem is proven. However, for the special case of 2-chromatic graphs the maximum contrast vector has all components equal to 1 trivially since a 2-colouring with extreme tones is precisely the maximum contrast greyscale. Nevertheless, in the present section we expose that the restricted version of this problem turns to be much more complicated within the same family. Therefore, throughout this section we study the $\{0,1\}$-RMACG problem for the family of bipartite graphs (equivalently 2-chromatic graphs) solely. It is well known that the detection of the bipartiteness of a graph with $n$ vertices has time complexity of order $\mathcal{O}\left(n^{2}\right)$. Thus, we will assume that for any bipartite graph its chromatic classes are given or, equivalently, a 2-colouring is known.

Let us observe that in case that $V_{c}$ contains adjacent vertices initially coloured with the same extreme tone, then the first component of the maximum contrast vector is equal to zero. In this particular case, the solution of the $\{0,1\}$-RMACG problem is 
essentially the same as in the case in which the edges with grey tone equal to zero are removed from the graph. Hence it is worth to focus on the problem with this extra condition of no adjacent vertices having the same initial colour in $V_{c}$. All along this section this condition is assumed without any further comment.

It suffices to revisit the proof of Lemma 1 to realize a similar result holds for the $\{0,1\}$-RMACG problem, included below in order to ease the reading.

Lemma 3 Let $G(V, E)$ be a connected graph and let $f$ be a maximum contrast greyscale compatible with an incomplete $V_{c}$-greyscale $g$. Let $v \in V-V_{c}$ be a vertex such that $0<f(v)<1$, then there exist $u_{1}$ and $u_{2} \in N(v)$ satisfying both of the following assertions:

1. $f\left(u_{1}\right)<f(v)<f\left(u_{2}\right)$.

2. $\widehat{f}\left(\left\{u_{1}, v\right\}\right)=\widehat{f}\left(\left\{u_{2}, v\right\}\right)=\min \{\widehat{f}(\{u, v\}): u \in N(v)\}$.

Recall that the vertices $u_{1}$ and $u_{2}$ given by Lemma 3, are referred as the pair of neighbours closest to $v ; u_{1}$ is the neighbour closest to $v$ on the left and $u_{2}$ is the neighbour closest to $v$ on the right.

The following proposition formulate a simple statement that will let us prove other results in a more elegant way.

Proposition 1 Let $G(V, E)$ be a connected graph and let $f$ be a maximum contrast greyscale compatible with an incomplete $V_{c}$-greyscale $g$. If there exists a vertex $v \in V$ such that $0<f(v)<1$, then the first component of $\mathcal{C}_{f}$ is at most $\frac{1}{2}$. Moreover, if the first component of $\mathcal{C}_{f}$ is equal to $\frac{1}{2}$, then $\operatorname{Im}(f)=\left\{0, \frac{1}{2}, 1\right\}$.

Proof If $f(v)$ is neither 0 nor 1 then, from Lemma 1, there exists a pair of neighbours closest to $v$, say $u_{1}$ and $u_{2}$, with $0 \leq f\left(u_{1}\right)<f(v)<f\left(u_{2}\right) \leq 1$ such that $f(v)-f\left(u_{1}\right)=f\left(u_{2}\right)-f(v)=\min _{u \in N(v)} \hat{f}(\{u, v\}) \geq \operatorname{lt}(G)$. Therefore, $1 \geq$ $f\left(u_{2}\right)-f\left(u_{1}\right) \geq 2 \operatorname{lt}(G)$ is held and then, $\operatorname{lt}(G) \leq \frac{1}{2}$. In the particular case that $\operatorname{lt}(G)=\frac{1}{2}$ we obtain $f\left(u_{2}\right)-f\left(u_{1}\right)=1$, which provides necessarily $f\left(u_{1}\right)=$ $0, f\left(u_{2}\right)=1$ and $f(v)=\frac{1}{2}$. Consequently, we reach $\operatorname{Im}(f)=\left\{0, \frac{1}{2}, 1\right\}$.

Let us fix some notation that will be used through this section. Any 2-chromatic graph $G$ has precisely two 2-colourings, say $\phi$ and $\bar{\phi}$, both using colours 0 and 1 such that for any vertex $v \in V, \bar{\phi}(v)=1-\phi(v)$. This way, for a given incomplete $V_{c}$-greyscale $g$, the set $V_{c}$ can be partitioned in two subsets $A \cup \bar{A}$, being $A$ the set of vertices of $V_{c}$ whose colour coincides with the colour assigned by $\phi$ and $\bar{A}=V_{c}-A$ being the vertices of $V_{c}$ whose colour coincides with the colour assigned by $\bar{\phi}$.

Remark 1 With the notation introduced above, if $f$ is a maximum contrast greyscale for the $\{0,1\}$-RMACG problem on a 2 -chromatic graph $G$, it is readily observed that the following statements are equivalent:

1. $V_{c}=A$ (equivalently $V_{c}=\bar{A}$ )

2. $f=\phi(f=\bar{\phi}$, respectively $)$

3. $\operatorname{Im}(f)=\{0,1\}$,

4. The contrast vector $\mathcal{C}_{f}$ has every component equal to 1 . 
We denote $P_{u v}$ the $u-v$ path in $G$, that is a path joining the vertices $u$ and $v$ as it is usually collected in the literature [see Harary (1969)].

Lemma 4 Let $f$ be a maximum contrast greyscale for the $\{0,1\}$-RMACG problem on a 2-chromatic connected graph $G$ and let $\phi$ be any 2-colouring of $G$. Then, for any pair of vertices $u \in A, v \in \bar{A}$ and for any $u-v$ path in $G$ there is a vertex $w$ in this path such that $f(w) \notin\{0,1\}$.

Proof Let $P_{u v}$ be a $u-v$ path in $G$ with length $l$. Since $G$ is a bipartite graph, for the 2-colouring $\phi$ it is verified that either $l$ is an even number if and only if $\phi(u)=\phi(v)$ or else $l$ is an odd number if and only if $\phi_{i}(u) \neq \phi(v)$, for $i=0,1$. Therefore, for the greyscale $f$ there are only two possibilities: either $f(u)=f(v)$ and $l$ is odd or else $f(u) \neq f(v)$ and $l$ is even. It is straightforwardly checked that there is a vertex $w \in P_{u v}$ such that $f(w) \notin\{0,1\}$, since otherwise, the first component of the maximum contrast vector $\mathcal{C}_{f}$ is 0 .

The following result provides the set of all possible values that a maximum contrast greyscale for the $\{0,1\}$-RMACG problem on the family of 2-chromatic graphs can reach.

Theorem 6 Let $G(V, E)$ be a 2-chromatic connected graph and let $f$ be a maximum contrast greyscale for the $\{0,1\}-\mathrm{RMACG}$ problem on $G$. Then, it is verified that $\operatorname{Im}(f) \subseteq\left\{0, \frac{1}{3}, \frac{1}{2}, \frac{2}{3}, 1\right\}$.

Proof Let us consider the set of initially coloured vertices $V_{c} \subset V$ with incomplete greyscale $g$ and any fixed 2-colouring on $G, \phi: V \rightarrow\{0,1\}$. From Remark 1, we may assume that the non-trivial cases satisfying $A \neq \emptyset$ and $\bar{A} \neq \varnothing$ are the ones that need to be analysed.

We define the following greyscale on $G$ :

$$
f_{\phi}(v)= \begin{cases}g(v) & \text { if } v \in V_{c} \\ \frac{2}{3} & \text { if } v \notin V_{c} \text { and there is } u \in V_{c} \cap N(v) \text { and } \phi(u) \neq g(u)=0 \\ \frac{1}{3} & \text { if } v \notin V_{c} \text { and there is } u \in V_{c} \cap N(v) \text { and } \phi(u) \neq g(u)=1 \\ & \text { and there is no } u \in V_{c} \cap N(v) \text { such that } \phi(u) \neq g(u)=0 \\ \phi(v) & \text { otherwise. }\end{cases}
$$

It is not difficult to check that $f_{\phi}$ is well defined and compatible with $g$. Besides, since $G$ is bipartite and equivalently it has no odd cycles, $\operatorname{Im}\left(\widehat{f_{\phi}}\right) \subseteq\left\{\frac{1}{3}, \frac{2}{3}, 1\right\}$ holds. Hence, the contrast vector $\mathcal{C}_{f_{\phi}}$ has first component $\frac{1}{3}$ and, therefore, the maximum contrast vector compatible with $g$ must have first component $a \geq \frac{1}{3}$.

Assume that $f$ is a maximum contrast greyscale compatible with $g$ and let us suppose that there is a vertex $u \in V$ such that $f(u) \notin\left\{0, \frac{1}{3}, \frac{1}{2}, \frac{2}{3}, 1\right\}$. From Lemma 3 , there exists the pair of neighbours closest to $u, u_{1}$ and $u_{2}$, such that $f\left(u_{1}\right)<$ $f(u)<f\left(u_{2}\right)$ and $f(u)-f\left(u_{1}\right)=f\left(u_{2}\right)-f(u)=d$. We analyse the value $d$. Observe that $d<\frac{1}{2}$, since $d=\frac{1}{2}$ implies $f(u)=\frac{1}{2}$ which is ruled out. Nonetheless, $\widehat{f}\left(\left\{u, u_{1}\right\}\right)=d \geq a \geq \frac{1}{3}$, thus $\frac{1}{3} \leq d<\frac{1}{2}$. 

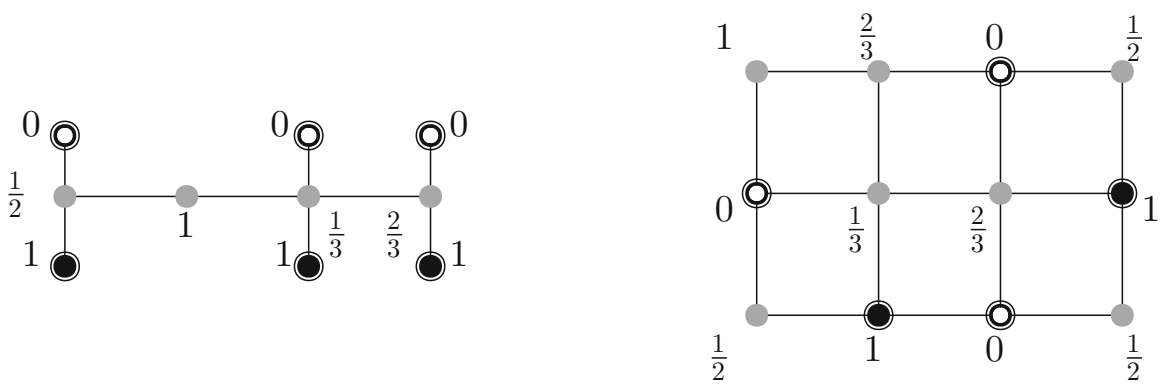

Fig. 4 Maximum contrast greyscales for the $\{0,1\}$-RMACG problem on this tree and this grid are given. Double circles in vertices denote the set $V_{c}$ of initially coloured vertices

Since $d<\frac{1}{2}$, it is clear that $f\left(u_{1}\right) \neq 0$ or $f\left(u_{2}\right) \neq 1$. Let us suppose $f\left(u_{1}\right) \neq 0$ and consider the pair of neighbours closest to $u_{1}$, namely $u_{3}$ and $u_{4}$, given by Lemma 3 . Then, $f\left(u_{3}\right)<f\left(u_{1}\right)<f\left(u_{4}\right)$ and $\frac{1}{3} \leq f\left(u_{1}\right)-f\left(u_{3}\right)=f\left(u_{4}\right)-f\left(u_{1}\right) \leq d$. Therefore $f\left(u_{2}\right)-f\left(u_{3}\right) \geq \frac{1}{3}+2 d \geq 1$, which implies $d=\frac{1}{3}$ and $f(u)=\frac{2}{3}$ contradicting our assumption. In a similar manner, if $f\left(u_{2}\right) \neq 1$ we reach $f(u)=\frac{1}{3}$ which is also impossible.

Then, we conclude $\operatorname{Im}(f) \subseteq\left\{0, \frac{1}{3}, \frac{1}{2}, \frac{2}{3}, 1\right\}$ and the proof is finished.

We want to emphasize that the bound set given by Theorem 6 is tight in the sense that some 2-chromatic connected graphs for which $\operatorname{Im}(f)=\left\{0, \frac{1}{3}, \frac{1}{2}, \frac{2}{3}, 1\right\}$ can be found. (See Fig. 4).

Next, we tackle the $\{0,1\}$-RMACG problem in three families of bipartite graphs with some constrains in the set $A$ in such a way that $\operatorname{Im}(f)=\left\{0, \frac{1}{2}, 1\right\}$ for the corresponding maximum contrast greyscale $f$. Recall that from its definition, when $\operatorname{Im}(f)=\left\{0, \frac{1}{2}, 1\right\}, \mathcal{C}_{f}$ has as few components equal to $\frac{1}{2}$ as possible. The proofs of the following results include the methods for assigning the greytone $\frac{1}{2}$ to the suitable vertices in order to obtain the maximum contrast greyscale $f$.

Theorem 7 The $\{0,1\}$-RMACG problem on any complete bipartite graph with an incomplete $V_{c}$-incomplete greyscale is solved with a computational complexity of order $\mathcal{O}\left(\left|V_{c}\right| n\right)$, where $n$ is the number of vertices of $G$. The maximum contrast vector has either all components equal to 1 or else all components equal to $\frac{1}{2}$.

Proof Let $f$ be a maximum contrast greyscale compatible with an incomplete $V_{c^{-}}$ greyscale on the complete bipartite graph $K_{r, s}$. We can assume $\operatorname{Im}(f) \neq\{0,1\}$, otherwise Remark 1 yields $\operatorname{Im}(f)=\{0,1\}$ and $\mathcal{C}_{f}$ has all components equal to 1 . Consider any fixed 2-colouring $\phi$ on $K_{r, s}$. Since $V_{c}$ has no adjacent vertices with the same initial extreme tone and by the assumption, the partition $V_{c}=A \cup \bar{A}$ verifies $A \neq \emptyset$ and $\bar{A} \neq \varnothing$.

From the completeness of $K_{r, s}$, it is deduced that $V_{c}$ must be a subset of one of the chromatic classes of the graph $K_{r, s}$. Therefore, all vertices of the other chromatic class of $K_{r, s}$ must have assigned the grey tone $\frac{1}{2}$ in order to give a maximum contrast greyscale for the $\{0,1\}$-RMACG problem on $K_{r, s}$. The remaining uncoloured vertices 
can be coloured using any extreme tone. Hence, its maximum contrast vector has all the components equal to $\frac{1}{2}$.

The computational cost is obtained by checking the chromatic class to which each vertex $v \in V_{c}$ belongs.

Immediately, a characterization of the case of 2-chromatic connected graphs with $A$ or $\bar{A}$ having precisely one vertex is presented.

Theorem 8 Let $f$ be a maximum contrast greyscale for the $\{0,1\}$-RMACG problem on a 2-chromatic connected graph $G$ and let $\phi$ be any 2-colouring of $G$, with $|A|=1$ and $|\bar{A}| \geq 1$. Then, it is verified that $\operatorname{Im}(f)=\left\{0, \frac{1}{2}, 1\right\}$.

Proof Let $f$ be a maximum contrast greyscale compatible with an incomplete $V_{c^{-}}$ greyscale $g$ of $G$ and $A=\left\{v_{0}\right\}$. From Lemma 4, $f$ takes at least a grey tone different from 0 and 1, and from Proposition 1, the first component of the vector $\mathcal{C}_{f}$ is at most $\frac{1}{2}$.

Next, let us define the following greyscale:

$$
f_{0}(v)= \begin{cases}g(v) & \text { if } v \in V_{c} \\ \frac{1}{2} & \text { if } v \in N\left(v_{0}\right) \\ \phi(v) & \text { otherwise. }\end{cases}
$$

It is checked that $f_{0}$ is well defined. In particular, no pair of adjacent vertices are coloured with the same grey tone since there are no triangles in the subgraph induced by $N\left(v_{0}\right)$ and $\phi$ is a proper colouring on $V-N\left(v_{0}\right)$. Hence, the first component of the contrast vector $\mathcal{C}_{f_{0}}$ is $\frac{1}{2}$. Therefore, since $\mathcal{C}_{f} \geq \mathcal{C}_{f_{0}}$, then $\mathcal{C}_{f}$ has its first component equal to $\frac{1}{2}$. Finally, from Proposition 1 we reach $\operatorname{Im}(f)=\left\{0, \frac{1}{2}, 1\right\}$.

As another interesting example, we show the computational complexity of the $\{0,1\}$-RMACG problem in the family of subdivided star graphs with any set of initially coloured leaves.

Theorem 9 Let $T$ be a subdivision of the star graph $K_{1, n}$ where $\left\{v_{1}, \ldots, v_{n}\right\}$ for $n \geq 3$, denote its leaves, $u$ the vertex of degree $n$ in $T$ and $g$ an incomplete greyscale such that $g\left(v_{i}\right) \in\{0,1\}$, for $1 \leq i \leq n$. The $\{0,1\}$-RMACG problem on $T$ is solved with a computational complexity of order $\mathcal{O}\left(\left|V_{c}\right| n\right)$.

Moreover, any maximum contrast greyscale $f$ uses precisely the grey tone $\frac{1}{2}$ over at most $\left\lfloor\frac{n}{2}\right\rfloor$ vertices, each one of them lying in a different path $P_{u v_{i}}$ and the maximum contrast vector $\mathcal{C}_{f}$ has at most $n$ components equal to $\frac{1}{2}$.

Proof Firstly, let $\phi$ be any 2-colouring of $T$. From Remark 1 we may assume that $\operatorname{Im}(f) \neq\{0,1\}$, otherwise the statement holds.

Next, let us observe that since the paths $P_{u v_{i}}$ intersect only in the vertex $u$, we can define a greyscale $f^{\prime}$ that assigns $f^{\prime}(u)=\frac{1}{2}, f^{\prime}\left(v_{i}\right)=g\left(v_{i}\right)$ and extends this colouring for each $P_{u v_{i}}$ by starting at $v_{i}$ and alternates 0 and 1 until the vertices of $N(u)$ are reached. $\mathcal{C}_{f^{\prime}}$ has precisely its $n$ first components equal to $\frac{1}{2}$ and the rest of 
components are equal to 1 . Hence, as $\mathcal{C}_{f} \geq \mathcal{C}_{f^{\prime}}$, then $\mathcal{C}_{f}$ has at most $n$ components equal to $\frac{1}{2}$.

Moreover, if $f(u)=\frac{1}{2}$, then $\mathcal{C}_{f^{\prime}}=\mathcal{C}_{f}$, therefore $u$ is the only vertex with grey tone equal to $\frac{1}{2}$. We reach to the assertion since $\left\lfloor\frac{n}{2}\right\rfloor \geq 1$.

Now, let us consider the case in which $f(u) \neq \frac{1}{2}$. Without loss of generality we may suppose $\phi(u)=0$. Let us denote $n_{0}$ the number of paths $P_{u v_{i}}$ verifying $g\left(v_{i}\right)=\phi\left(v_{i}\right)$ and let us denote $n_{1}$ the number of paths $P_{u v_{i}}$ verifying $g\left(v_{i}\right)=\bar{\phi}\left(v_{i}\right)$. From the initial assumption, $n_{i} \neq 0$ for $i=0,1$.

Next, by selecting the colour $c$ for $u$ such that $n_{c}=\max \left\{n_{0}, n_{1}\right\}$ and from the classical pigeonhole principle, it is deduced that $n_{c} \geq\left\lceil\frac{n}{2}\right\rceil$. Let $\phi_{c}$ be the appropriately selected coloring $\phi$ or $\bar{\phi}$.

Finally, let us define $f^{\prime \prime}$ a greyscale compatible with $g$ in the following way:

$$
f^{\prime \prime}(v)=\left\{\begin{array}{cl}
g(v) & \text { if } v=v_{i} \text { for } i=1, \ldots, n \\
\frac{1}{2} & \text { if } v \in N\left(v_{i}\right) \text { for each vertex } v_{i} \text { such that } \phi_{c}\left(v_{i}\right) \neq g\left(v_{i}\right) \\
\phi_{c}(v) & \text { in other case. }
\end{array}\right.
$$

This way, only a vertex of $P_{u v_{i}}$ has grey tone $\frac{1}{2}$, for at most $n-\left\lceil\frac{n}{2}\right\rceil=\left\lfloor\frac{n}{2}\right\rfloor$ paths $P_{u v_{i}}$. We conclude that since the maximum contrast greyscale compatible with $g, f$, verifies $\mathcal{C}_{f} \geq \mathcal{C}_{f^{\prime \prime}}$, the assertion holds. The computational cost is obtained by checking the chromatic class to which each vertex $v_{i} \in V_{c}$ belongs and by the comparison between $f^{\prime}$ and $f^{\prime \prime}$.

Now, we deal with the family of trees with precisely three initially coloured vertices. The relevance of the next result is based on the method of assigning the grey tone $\frac{1}{2}$ which is described in the proof.

Theorem 10 The $\{0,1\}$-RMACG problem on a tree $T=G(V, E)$ with an incomplete $V_{c}$-greyscale $g$ and $\left|V_{c}\right|=3$ is solved with a computational complexity of order $\mathcal{O}\left(n^{2}\right)$. Moreover, if $f$ is a maximum contrast greyscale for the $\{0,1\}$-RMACG problem then it uses the greytone $\frac{1}{2}$ over at most 2 vertices.

Proof Let $V_{c}=\left\{v_{1}, v_{2}, v_{3}\right\} \subset V$ and let us consider the 2-colourings $\phi$ and $\bar{\phi}$ on $T$. From Remark 1 we may assume $\operatorname{Im}(f) \neq\{0,1\}$. Without loss of generality we may suppose $|A|=2$. Recall that $|A|=3$ implies $\operatorname{Im}(f)=\{0,1\}$. From Theorem 8 , we get $\operatorname{Im}(f)=\left\{0, \frac{1}{2}, 1\right\}$. Moreover, the number of components equal to $\frac{1}{2}$ in the maximum contrast vector $\mathcal{C}_{f}$ coincides with the number of vertices adjacent to those vertices coloured with the grey tone $\frac{1}{2}$, that is the sum of the degrees of all vertices coloured with $\frac{1}{2}$. Without loss of generality, let us suppose $\phi\left(v_{1}\right)=g\left(v_{1}\right)$, $\phi\left(v_{2}\right)=g\left(v_{2}\right)$ and $\phi\left(v_{3}\right) \neq g\left(v_{3}\right)$. By Lemma 4 , there is a vertex $w_{1} \in P_{v_{1} v_{3}}$ and a vertex $w_{2} \in P_{v_{2} v_{3}}$ with $f\left(w_{1}\right)=f\left(w_{2}\right)=\frac{1}{2}$. Since $f$ is a maximum contrast greyscale compatible with $g$ on $T$, the restriction of $f$ to the union of the three paths $P_{v_{i} v_{j}}$, for $1 \leq i<j \leq 3$, assigns the grey tone $\frac{1}{2}$ only to $w_{1}$ and $w_{2}$. Due to the fact that $T$ has no cycles, it is straightforwardly checked that, by starting from each $v_{i} \in V_{c}, f$ assigns 0 and 1 appropriately to the remaining vertices of $T-\left\{V_{c} \cup\left\{w_{1}, w_{2}\right\}\right\}$. Hence, $\left\{w_{1}, w_{2}\right\}$ are the only vertices with grey tone $\frac{1}{2}$. 
More precisely, let $W$ be the set of vertices within $V\left(P_{v_{1} v_{3}} \cup P_{v_{2} v_{3}} \cup P_{v_{1} v_{2}}\right)-V_{c}$ with minimum degree in $T$. If there exists a vertex $w \in W$ such as $w \in V\left(P_{v_{1} v_{3}}\right) \cap V\left(P_{v_{2} v_{3}}\right)$, then $w_{1}=w_{2}=w$ and $f$ assigns the grey tone $\frac{1}{2}$ to precisely one of such a vertex $w$. Otherwise, without loss of generality, let us suppose $w \in V\left(P_{v_{1} v_{3}}\right)-V\left(P_{v_{2} v_{3}}\right)$ and consider a pair of vertices $w^{\prime} \in V\left(P_{v_{1} v_{3}}\right) \cap V\left(P_{v_{2} v_{3}}\right)$ and $w^{\prime \prime} \in V\left(P_{v_{2} v_{3}}\right)-V\left(P_{v_{1} v_{3}}\right)$ both with minimum degree in $T$. If $\operatorname{deg}\left(w^{\prime}\right) \leq \operatorname{deg}(w)+\operatorname{deg}\left(w^{\prime \prime}\right)$, then $w_{1}=w_{2}=$ $w^{\prime}$ and $f$ assigns the grey tone $\frac{1}{2}$ to precisely one of such a vertex $w^{\prime}$. If not, $f$ assigns $\frac{1}{2}$ to precisely $w_{1}=w$ and $w_{2}=w^{\prime \prime}$ or to another pair of vertices $w$ and $w^{\prime \prime}$ verifying the same conditions as $w_{1}$ and $w_{2}$, respectively. The computational cost is obtained by computing the three paths and the vertices within them that have minimum degree in the graph.

We observe that finding the solution of the $\{0,1\}$-RMACG problem on bipartite graphs may become complicated in the general case.

In fact, we have given several examples showing that there are trees with few vertices that need precisely the full set of 5 grey tones given by Theorem 6 (see Fig. 4). Many other examples of trees with similar properties may be found. Hence, finding the solution of the $\{0,1\}$-RMACG problem is not an easy question to answer even for the case of trees.

In a future research, we would like to discover the nature of this problem, whether it is an NP-complete one or not.

\section{Results and open questions}

We have introduced the new concept of the contrast of a graph related to vertex and edge colourings that use colours from the continuous spectrum $[0,1]$. The minimum colour difference between adjacent vertices of the graph plays a fundamental role in the maximum contrast problem (MACG problem) and its close relation with the chromatic number of the graph has been proven. As a straightforward consequence of this fact, the NP-completeness of the MACG problem is established (Theorem 1) and a new invariant of the graph has been found. Otherwise, Theorem 2 presents results regarding the underlying structure of the colour differences between adjacent vertices.

We have achieved several results that allow us to compute the set of all possible values of grey tones for maximum contrast greyscales of graphs with known chromatic number. Moreover, some notions of subsets in $[0,1]$ are introduced, such as the $h$ minimum-step-enchained set. We want to remark here that this kind of sets may be considered independently from any graph and could be useful in other branches of Mathematics. By using the algorithmic procedure MAXIMAL ENCHAINED SET included in this paper, we can prove that the set of possible values of grey tones of a maximum contrast greyscale of a graph is a rational finite set (Theorem 5).

Some natural questions remain open. The properties established in Theorems 2 and 5 can be useful to develop approximation algorithms for the NP-complete MACG problem, and, furthermore, it is interesting to tackle the contrast problem for particular families of graphs. 
Nonetheless, we give another version of the MACG problem consisting of solving the same question but initially assigning some extreme tones to a subset of vertices. We name it $\{0,1\}$-Restricted maximum contrast on graphs, $\{0,1\}$-RMACG problem in short. We have provided a bound set of values of a solution for the $\{0,1\}$-RMACG problem on any bipartite graph (Theorem 6). The particular case of the $\{0,1\}$-RMACG problem on bipartite complete graphs is solved (Theorem 7) and it is solved as well for particular family of trees with additional conditions on the set of vertices initially coloured (Theorems 8 and 10). However, another improvement of these results like solving the problem in other families of graphs such as outerplanar graphs or planar graphs will be dealt with future works. Besides, another open problem appears if we change the restriction of initially coloured vertices with extreme tones $\{0,1\}$ and consider any other grey tones for the incomplete greyscale.

Furthermore, based on the results of different computational simulations that we have carried out, another interesting line of work consists of obtaining good approximations for the optimal solution of the antibandwidth problem from maximum contrast greyscales. Particularly, suitable vertex labelings are achieved when the graph vertices are sorted following an increasing order of their greytones for a maximum contrast greyscale.

Lastly, and in addition to the relations of the MACG problem to other well-known combinatorial optimization problems pointed out along this paper, we would like to highlight that the interest of these new concepts relies in their possible application in the solution of problems in engineering, physics and applied mathematics, which are modelled according to a network whose nodes have assigned numerical values of a certain parameter delimited by a range of real numbers. The objective is to maximize the differences between each node and its neighbours, from a local and global point of view simultaneously through a vectorial objective function, that is the contrast vector.

Acknowledgements The authors gratefully acknowledge financial support by the Spanish Ministerio de Economía, Industria y Competitividad and Junta de Andalucía via Grants, MTM2015-65397-P (M.T. VillarLiñán) and PAI FQM-164, respectively. The authors also thank two anonymous referees for their insightful reports that have helped us improve the paper substantially.

\section{References}

Aardal KI, van Hoesel SPM, Koster AMCA, Mannino C, Sassano A (2007) Models and solution techniques for frequency assignment problems. Ann Oper Res 153(1):79

Bekos MA, Kaufmann M, Kobourov SG, Stavropoulos K, Veeramoni S (2017) The maximum k-differential coloring problem. J Discrete Algorithms 45:35-53

Borghini F, Méndez-Díaz I, Zabala P (2018) An exact algorithm for the edge coloring by total labeling problem. Ann Oper Res. https://doi.org/10.1007/s10479-018-2977-x

Bullnheimer B (1998) An examination scheduling model to maximize students' study time. Lect Notes Comput Sci 1408:78-91

Chaitin GJ, Auslander MA, Chandra AK, Cocke J, Hopkins ME, Markstein PW (1981) Register allocation via coloring. Comput Lang 6(1):47-57

Chaudhry AU, Chinneck JW, Hafez RHM (2016) Fast heuristics for the frequency channel assignment problem in multi-hop wireless networks. Eur J Oper Res 251(3):771-782

Cheng W-T, Lewandowski JL, Wu E (1992) Optimal diagnostic methods for wiring interconnects. IEEE Trans Comput Aided Des Integr Circuits Syst 11(9):1161-1166 
de Castro N, Garrido-Vizuete MA, Robles R, Villar MT (2016) The maximal $\frac{1}{k}$-minimum-step-enchained sets $F_{k}$, for $k=2, \ldots 7$. https://www.researchgate.net/publication/311576608_FksetsContrast. https://doi.org/10.13140/RG.2.2.11475.78887

de Castro N, Garrido-Vizuete MA, Robles R, Villar MT (2018) Gradation in grayscale of graphs. arXiv, Cornell University e-print repository, paper no. arXiv:1612.07552v5

Demange M, Ekim T, Ries B, Tanasescu C (2015) On some applications of the selective graph coloring problem. Eur J Oper Res 240(2):307-314

Dillencourt Michael B, Eppstein D, Goodrich MT (2007) Choosing colors for geometric graphs via color space embeddings. In: Kaufmann M, Wagner D (eds) Procedings 14th international symposium on graph drawing (GD'06), volume 4372 of lecture notes in computer science. Springer, pp 294-305

Ganguli R, Roy S (2017) A study on course timetable scheduling using graph coloring approach. Int J Comput Appl Math 12(2):469-485

Garey MR, Johnson DS (1979) In computers and intractability: a guide to the theory of NP-completeness. WH Freeman and company, San Francisco

Griggs JR, Král D (2009) Graph labellings with variable weights, a survey. Discrete Appl Math 157(12):2646-2658

Harary F (1969) In graph theory. Addison-Wesley, Boston

Hu Y, Kobourov S, Veeramoni S (2011) On maximum differential graph coloring. In: Brandes U, Cornelsen S (eds) Proceedings of 18th international symposium on graph drawing (GD'10), volume 6502 of lecture notes in computer science. Springer, pp 274-286

Karp RM (1972) Reducibility among combinatorial problems. In: Miller RE, Thatcher JW (eds) Complexity of computer computations. Springer, New York, pp 85-103

Kasi SK, Naqvi IH, Kasi MK, Yaseen F (2019) Interference management in dense inband D2D network using spectral clustering \& dynamic resource allocation. Wireless Netw 25:4431-4441. https://doi. org/10.1007/s11276-019-02107-2

Lecat C, Lucet C, Li C-M (2017) Minimum sum coloring problem: upper bounds for the chromatic strength. Discrete Appl Math 233:71-82

Leung JYT, Vornberger O, Witthoff JD (1984) On some variants of the bandwidth minimization problem. SIAM J Comput 13(3):650-667

Marx D (2004) Graph colouring problems and their applications in scheduling. Electr Eng 48(1-2):11-16

Orden D, Marsa-Maestre I, Giménez-Guzmán JM, de la Hoz E (2016) Bounds on spectrum graph coloring. Electron Notes Discrete Math 54:63-68

Orden D, Giménez-Guzmán JM, Marsa-Maestre I, De la Hoz E (2018) Spectrum graph coloring and applications to Wi-Fi channel assignment. Symmetry 10(3):65

Orden D, Marsa-Maestre I, Giménez-Guzmán JM, de la Hoz E, Álvarez-Suárez A (2019) Spectrum graph coloring to improve Wi-Fi channel assignment in a real-world scenario via edge contraction. Discrete Appl Math 263:234-243

Woo T-K, Su SY, Newman-Wolfe R (1991) Resource allocation in a dynamically partitionable bus network using a graph coloring algorithm. IEEE Trans Commun 39(12):1794-1801

Publisher's Note Springer Nature remains neutral with regard to jurisdictional claims in published maps and institutional affiliations. 